\title{
Dynamic tracking of microvascular hemoglobin content for continuous perfusion monitoring in the intensive care unit: pilot feasibility study
}

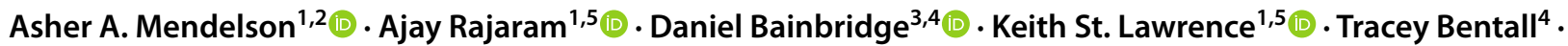 \\ Michael Sharpe ${ }^{3,4} \cdot$ Mamadou Diop ${ }^{1,5} \cdot$ Christopher G. Ellis ${ }^{1,2,6} \cdot$ On behalf of the Canadian Critical Care \\ Translational Biology Group
}

Received: 17 July 2020 / Accepted: 20 October 2020 / Published online: 26 October 2020

(c) Springer Nature B.V. 2020

\begin{abstract}
Purpose: There is a need for bedside methods to monitor oxygen delivery in the microcirculation. Near-infrared spectroscopy commonly measures tissue oxygen saturation, but does not reflect the time-dependent variability of microvascular hemoglobin content (MHC) that attempts to match oxygen supply with demand. The objective of this study is to determine the feasibility of MHC monitoring in critically ill patients using high-resolution near-infrared spectroscopy to assess perfusion in the peripheral microcirculation. Methods: Prospective observational cohort of 36 patients admitted within $48 \mathrm{~h}$ at a tertiary intensive care unit. Perfusion was measured on the quadriceps, biceps, and/or deltoid, using the temporal change in optical density at the isosbestic wavelength of hemoglobin $(798 \mathrm{~nm})$. Continuous wavelet transform was applied to the hemoglobin signal to delineate frequency ranges corresponding to physiological oscillations in the cardiovascular system. Results: $31 / 36$ patients had adequate signal quality for analysis, most commonly affected by motion artifacts. MHC signal demonstrates inter-subject heterogeneity in the cohort, indicated by different patterns of variability and frequency composition. Signal characteristics were concordant between muscle groups in the same patient, and correlated with systemic hemoglobin levels and oxygen saturation. Signal power was lower for patients receiving vasopressors, but not correlated with mean arterial pressure. Mechanical ventilation directly impacts MHC in peripheral tissue. Conclusion: MHC can be measured continuously in the ICU with high-resolution near-infrared spectroscopy, and reflects the dynamic variability of hemoglobin distribution in the microcirculation. Results suggest this novel hemodynamic metric should be further evaluated for diagnosing microvascular dysfunction and monitoring peripheral perfusion.
\end{abstract}

Keywords Microcirculation $\cdot$ Near-infrared spectroscopy $\cdot$ Hemodynamic monitor $\cdot$ Critical care $\cdot$ Wavelet analysis

\section{Introduction}

Electronic supplementary material The online version of this article (https://doi.org/10.1007/s10877-020-00611-x) contains supplementary material, which is available to authorized users.

Christopher G. Ellis

cgellis@uwo.ca

1 Department of Medical Biophysics, Western University, London, ON, Canada

2 Centre for Critical Illness Research, Lawson Health Research Institute, London, ON, Canada

3 Department of Anesthesia and Peri-operative Medicine, Western University, London, ON, Canada
The peripheral microcirculation is one of the physiological windows that can be assessed at the bedside for patients in the intensive care unit (ICU) with circulatory shock

4 Division of Critical Care, Department of Medicine, Western University, London, ON, Canada

5 Imaging Program, Lawson Health Research Institute, London, ON, Canada

6 Robarts Research Institute, Rm 3205, London, ON N6A 5B7, Canada 
[1]. Perfusion status correlates better with ICU outcomes than macro-hemodynamics [2,3], and there is emerging study of perfusion-guided resuscitation using clinical indicators such as capillary refill time, skin mottling, and body temperature gradients $[4,5]$. Although these measurements can be standardized, they are often subject to inter-observer variability and lack refinement beyond rudimentary measures.

Microvascular monitoring has been proposed as an adjunct strategy for quantifying peripheral perfusion in the ICU $[6,7]$. Near-infrared spectroscopy (NIRS) can be applied continuously and non-invasively to measure tissue oxygen saturation $\left(\mathrm{StO}_{2}\right)$ of hemoglobin in skeletal muscle and overlying tissue [8]. Since venules contain the majority of blood in the microcirculation, $\mathrm{StO}_{2}$ can be viewed primarily as a measure of regional venous oxygen saturation [9]. However, a recent randomized controlled trial did not show benefit for incorporating $\mathrm{StO}_{2}$ in a sepsis protocol [10], suggesting this may not be the correct target for microvascular resuscitation.

There is a need for physiologically-informed methods for evaluating the peripheral microcirculation that describe the dynamic process of oxygen delivery in microvascular networks. Traditionally, NIRS considers two distinct hemoglobin chromophores: oxy- $\left(\mathrm{Hb}-\mathrm{O}_{2}\right)$ and deoxy(Hb) hemoglobin, with the sum of both chromophores denoted as total hemoglobin ( $\mathrm{HbT}$ ) [11]. Changes in $\mathrm{HbT}$ are proposed as a surrogate for changes in blood volume, assuming incorrectly that the microcirculation has a fixed hematocrit $[12,13]$. In reality, red blood cells (RBCs) do not exist in exclusively oxy/deoxy states and contain millions of hemoglobin proteins with an aggregate RBC oxygen saturation. Furthermore, hematocrit and RBC flow in microvascular networks are highly heterogeneous [14], and temporal variations in HbT reflect changes in blood volume, hematocrit, and flow velocity within all three microvascular compartments (arterioles, capillaries, and venules) $[8,15]$.

We propose microvascular hemoglobin content (MHC) to denote the total hemoglobin contained in all the RBCs in the microcirculation. Although technically equivalent to $\mathrm{HbT}$, the distinction arises from how this measurement is evaluated as a time-dependent physiological variable. MHC variability represents the continuous process of RBC distribution that is regulated within the underlying microcirculation, and we hypothesize that MHC may serve as a novel hemodynamic metric for monitoring microvascular function in the ICU. The goal of the current study is to (i) outline the theory and rationale for the technology, (ii) evaluate the feasibility of signal acquisition and signal analysis in the intended clinical environment, and (iii) present the findings of this pilot cohort with an emphasis on signal characterization and descriptive statistics.

\section{Methods}

\subsection{Study details}

This is a prospective observational cohort study. Patients were recruited in a tertiary adult medical-surgical ICU (July 2017-April 2019) and all research practices were performed in accordance with institutional ethics protocol and approval (REB \#108760). Participants were recruited within $48 \mathrm{~h}$ of admission to the ICU, and consent was obtained from the patient or substitute decision maker prior to commencement of the study. The NIRS system was applied to the quadriceps, biceps, and/or deltoid; additional protocol information can be found in supplementary content. This was a one-time observational study, with no additional follow-up or intervention other than MHC monitoring.

\subsection{High-resolution NIRS for measuring microvascular hemoglobin content}

Microvascular hemoglobin content (i.e. HbT concentration) is derived from NIRS signal sampled at high temporal resolution $(10 \mathrm{~Hz})$. For most NIRS wavelengths, variations in hemoglobin concentration (HbT) or saturation $\left(\mathrm{StO}_{2}\right)$ can both affect tissue absorption. The isosbestic wavelength of hemoglobin defines the wavelength with identical extinction coefficients for $\mathrm{Hb}-\mathrm{O}_{2}$ and $\mathrm{Hb}$; in the near-infrared spectrum this occurs at approximately $798 \mathrm{~nm}$ [16]. Therefore, changes in tissue absorbance at the isosbestic wavelength only detect variations in $\mathrm{HbT}$ concentration [11, 12]. In this study, MHC variability was measured using isosbestic NIRS, and can equally be calculated with two wavelengths to derive $\mathrm{HbT}$ as the sum of $\mathrm{Hb}-\mathrm{O} 2$ and $\mathrm{Hb}$ hemoglobin chromophores [12].

This time-dependent $\mathrm{HbT}$ measurement also accounts for the contribution of melanin and myoglobin, that significantly affect NIRS absorption in peripheral tissue [17]. Because melanin and myoglobin have fixed tissue concentrations, they do not contribute to the temporal variability in NIRS signal [8]; myoglobin saturation may vary within skeletal muscle, but the concentration is static. Thus, highresolution NIRS specifically measures the temporal change in hemoglobin content, as a surrogate for RBC flow within the underlying microcirculation.

\subsection{Engineering and system details}

Measurements were acquired with a continuous-wave broadband NIRS system based on a modified off-the-shelf 
spectrometer (QE65000 Ocean Optics, Dunedin, FL) and a halogen lamp (Ocean Optics HL-2000-HP). Technical specifications and proof-of-concept of the NIRS system have been described previously [18].

Data capture was performed using SpectraSuite ${ }^{\mathrm{TM}}$ software (Ocean Optics, Inc.). Light intensities were recorded at the isosbestic wavelength $798 \mathrm{~nm}$ with a sampling frequency of $10 \mathrm{~Hz}$ (100 ms integration time). Source detector distance (SDD) was $20 \mathrm{~mm}$ (7 participants) and $15 \mathrm{~mm}$ (29 participants). Shorter SDD interrogates a smaller volume of tissue and depth of penetration, but with higher light intensities recovered by the spectrometer; this modification was made when very low intensity signal was encountered for one patient early in the cohort.

Light intensity is converted to change in optical density $(\Delta \mathrm{OD})$ as follows:

$\Delta O D(t)=\log _{10} \frac{I\left(t_{o}\right)}{I(t)}$

where $\mathrm{I}\left(\mathrm{t}_{\mathrm{o}}\right)$ is the initial intensity at time $=0$, and $\mathrm{I}(\mathrm{t})$ is the intensity at any subsequent time, $t$.

Assuming that tissue scattering does not change during the recording, $\triangle \mathrm{OD}$ measures the change from baseline in $\mathrm{HbT}$ concentration in arbitrary units [19].

$\Delta \mathrm{OD}$ time series (i.e. MHC tracings) were screened manually for artifacts as described in supplementary content and supplementary Fig. 1. Only continuous tracings of greater than 5 min with adequate signal quality were included in the analysis.

\subsection{Continuous wavelet transform (CWT)}

Application of continuous wavelet transform (CWT) for analysis of cardiovascular time series was first described by Stefanovska and colleagues [20], and has gained widespread use in the field of laser doppler flowmetry (LDF) of human skin $[21,22]$. CWT is an attractive tool, as it provides good localization of signal in both time and frequency for nonstationary data. Using CWT, time series can be represented as power versus frequency versus time for physiological oscillations ascribed to components of the cardiovascular system [23]. Six "frequency bands" have been derived from pharmacological iontophoresis studies of human skin, and are proposed to represent mechanisms of peripheral and central blood flow control [21, 23-25] (Table 1): (1) B1 metabolic (2) B2 endothelial (3) B3 neurogenic (4) B4 myogenic (5) B5 respiratory (breath rate) and (6) B6 cardiac (heart rate).

CWT analysis was applied in this study to $\triangle \mathrm{OD}$ time series using the Morlet wavelet in Matlab v2016b (Fig. 1). The total power for each frequency band was computed as the sum over the frequency range. B1 (ultra-low frequency) and B6 (high frequency) were removed from the analysis as they were most susceptible to artifacts, as described in supplementary content. Thus, the final CWT signal is defined from B2-B5 and comprises three microvascular bands B2-B4 and the respiratory band B5. Using CWT, total signal power B2-B5 and for each frequency band is represented with mean, median, and coefficient of variation (\%CV) over the duration of the recording; median power was the metric most often used for analysis in this study, being less impacted by extreme values than the mean. When applicable, CWT measurements were averaged for each muscle site, and muscle sites were averaged to calculate summary values for each patient.

The percentage contribution (\%Power) of each frequency band relative to the total signal power was also quantified. For example, \%Power of the respiratory band B5 is calculated as:

$\%$ PowerB5 $=\frac{\text { median PowerB5 }}{\text { median PowerB2 }-B 5} \times 100$

This represents the contribution from the respiratory frequency range $(0.16-0.667 \mathrm{~Hz})$ to the total signal power. Similar calculations are used to quantify the contribution of frequency bands relative to the microvascular signal B2-B4.

\subsection{Statistical analysis}

Statistical analysis was performed using PRISM v8 software package. Lognormal distribution of median power values was assessed with D'Agostino Pearson test, with subsequent analysis performed using parametric tests on -logtransform of median power. Concordance between median power for frequency bands in the same patient was tested with a Pearson correlation matrix. Correlation between different muscle sites in the same patient was assessed with Pearson and Spearman correlation coefficient ( $r$ ) for median power and \%Power, respectively. MHC signal power was compared between patients requiring and not requiring vasoactive medication (vasopressors), patients with serum lactate greater or less than $2.0 \mathrm{mmol} / \mathrm{L}$, and between SDD configurations using Student's unpaired t-test; \%CV was compared between patients requiring and not requiring vasopressors using Mann-Whitney U test. Linear regression was used to calculate the coefficient of determination $\left(\mathrm{R}^{2}\right)$ between MHC signal power and systemic variables: mean arterial pressure (MAP), serum hemoglobin concentration ([Hb], $\mathrm{g} / \mathrm{L})$, arterial oxygen saturation $\left(\mathrm{SpO}_{2}, \%\right)$, and serum lactate $(\mathrm{mmol} / \mathrm{L})$.

Linear regression was used to assess correlation between MHC respiratory power B5 and \%Power B5, and ventilator 

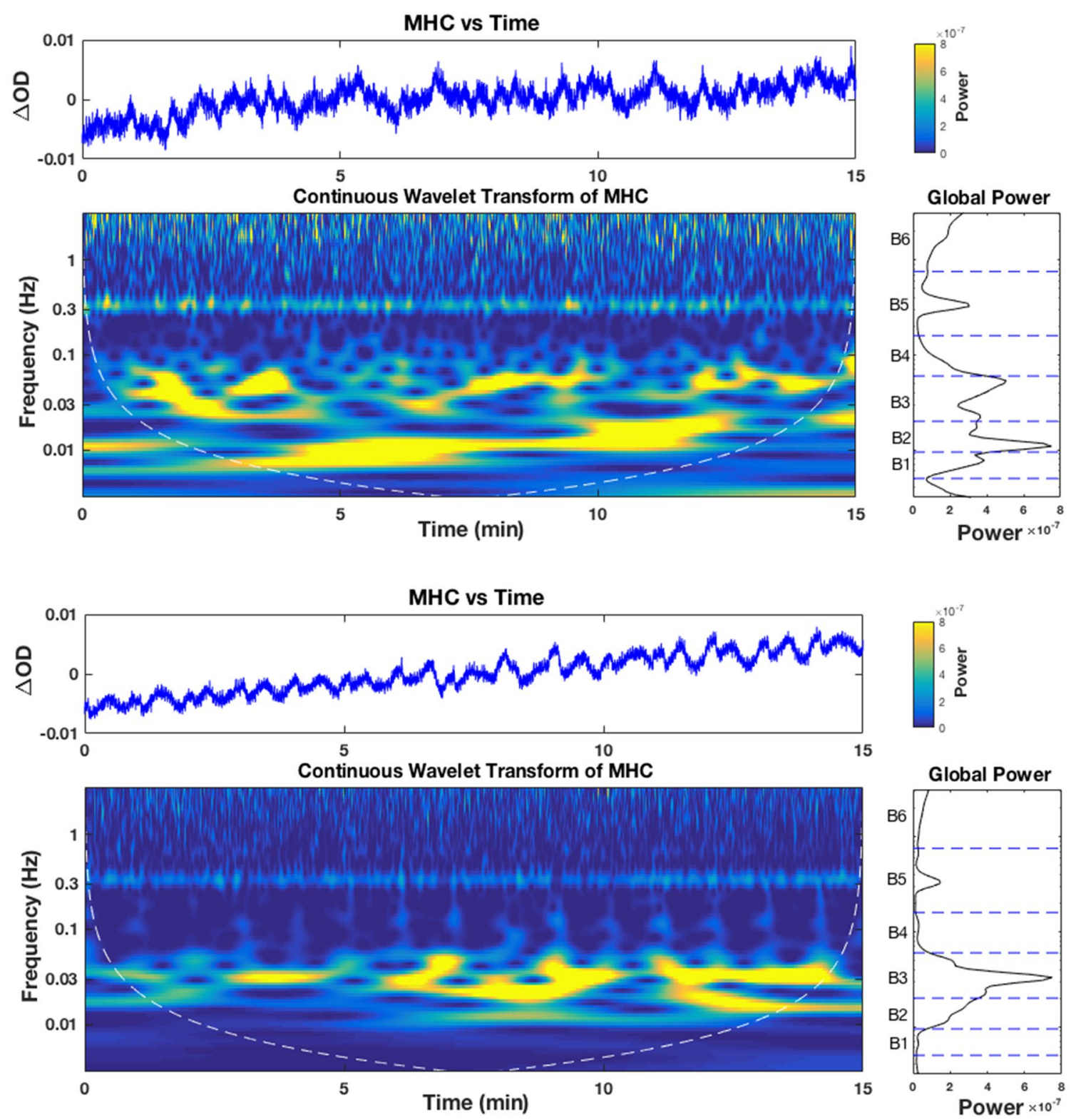

Fig. 1 Application of continuous wavelet transform (CWT) to microvascular hemoglobin content (MHC) time series in peripheral tissue for continuous perfusion monitoring. $15 \mathrm{~min}$ of data is shown for two patients with cardiac arrest (upper) and sepsis (lower). MHC variability is defined as the change from baseline in total hemoglobin concentration, measured at high temporal resolution $(10 \mathrm{~Hz})$ with isosbestic near-infrared spectroscopy ( $\Delta \mathrm{OD}$, arbitrary units). MHC time series demonstrates substantial temporal variability, as well as differences in variability patterns between patients. Data is represented with standard time series (MHC vs time), CWT time-frequency projection (power vs frequency vs time), and the global wavelet power spectrum (GWPS) averaged over the recording (power vs frequency). Dotted line in the CWT indicates data outside the cone of influence that is susceptible to edge effects. Dashed lines in the GWPS represent the boundaries of the frequency bands used for analysis in this study $(B I$ metabolic, $B 2$ endothelial, $B 3$ neurogenic, $B 4$ myogenic, $B 5$ respiratory, $B 6$ cardiac)

\section{Results}

\subsection{Feasibility metrics from the pilot cohort}

36 patients were recruited for the study with mean recording time $101.50 \pm 50.12 \mathrm{~min}$ (range: $0.00-221.10$ ) per 
Table 1 Frequency bands used in the present study, that are attributable to physiological oscillations in the cardiovascular system [23, 24, 43]

\begin{tabular}{llll}
\hline Frequency band & Frequency $(\mathrm{Hz})$ & Period (seconds) & Cycles/minute \\
\hline B0 & $0-0.005$ & $>200$ & $<0.3$ \\
B1-Metabolic & $0.005-0.0095$ & $105.3-200$ & $0.3-0.57$ \\
B2-Endothelial & $0.0095-0.02$ & $50-105.3$ & $0.57-1.2$ \\
B3-Neurogenic & $0.02-0.06$ & $16.67-50$ & $1.2-3.6$ \\
B4-Myogenic & $0.06-0.16$ & $6.25-16.67$ & $3.6-9.6$ \\
B5-Respiratory & $0.16-0.667$ & $1.5-6.25$ & $9.6-40$ \\
B6-Cardiac & $0.667-3.1$ & $0.32-1.5$ & $40-186$ \\
\hline
\end{tabular}

Frequency ranges are adapted from previous studies [21, 25], with modification to the upper limit of respiratory band to include rapid breathing rates observed in the ICU

Table 2 Demographics for the patients in the final dataset included for analysis $(n=31)$. Data are represented as mean $\pm S D$, median (IQR), or percentage of total cohort

\begin{tabular}{ll}
\hline & $\mathrm{N}=31$ patients \\
\hline Age (years) & $62.10 \pm 16.55$ \\
Male (\%) & 61.3 \\
Weight $(\mathrm{kg})$ & $82.12 \pm 17.67$ \\
$\mathrm{MAP}(\mathrm{mmHg})$ & $75.30 \pm 12.73$ \\
$\mathrm{HR}(\mathrm{bpm})$ & $83.32 \pm 15.68$ \\
Sepsis $(\%)$ & 35.5 \\
Cardiac arrest $(\%)$ & 19.4 \\
Vasopressors (\%) & 64.5 \\
$\mathrm{Mechanical} \mathrm{ventilation}(\%)$ FiO $_{2}$ & 90.3 \\
$\mathrm{SpO}_{2} \%$ & $0.40(0.2)$ \\
$\left.\mathrm{PEEP}_{(\mathrm{cm} \mathrm{H}} 0\right)$ & $97(3.5)$ \\
Lactate $(\mathrm{mmol} / \mathrm{L})$ & $10(4)$ \\
{$[\mathrm{Hb}](\mathrm{g} / \mathrm{L})$} & $1.4(0.8)$ \\
\hline
\end{tabular}

$M A P$ mean arterial pressure, $\mathrm{HR}$ heart rate, $\mathrm{FiO}_{2}$ fraction of inspired oxygen, $\mathrm{SpO}_{2}$ arterial oxygen saturation, $P E E P$ positive end-expiratory pressure, $[\mathrm{Hb}]$ systemic hemoglobin concentration

patient. After manually screening for signal quality and artifacts, the final data set comprised 31 patients with a mean recording time of $74.02 \pm 36.89 \mathrm{~min}$ (range: 5.83-148.70). Demographics for the final study cohort are provided in Table 2. Signal quality for the final cohort was high, with median 74\% (IQR 54-88) of recorded signal suitable for analysis; the most common reason for signal degradation was motion artifact. Of the five patients unsuitable for analysis, two were excluded due to measurements with compression stockings (this was a naïve error early in the study, but did demonstrate the venous occlusion waveform included in the supplementary content); one patient was excluded due to insufficient signal intensity (SDD of the NIRS system was subsequently modified, as described in methods); one patient was recruited but could not be measured due to clinical circumstances; and one patient was excluded due to excessive motion artifacts.

MHC tracings were recorded from 54 muscles with a mean duration of $42.49 \pm 22.34$ min (range: 5.50-93.66) per muscle. 20/31 patients had recordings from multiple muscle sites, including three patient with recordings from 3 muscle sites. The main limitations for measuring multiple muscle sites was the presence of compression stockings on the lower extremities, patient positioning, lines and tubing, and change in clinical condition. Vastus medialis and biceps were the most common muscle sites for recording, and easiest to measure with patient positioning and clinical workflow; the deltoid muscle site was most vulnerable to motion artifacts and vastus lateralis was suboptimal due to patient positioning.

\subsection{MHC signal demonstrates non-stationary temporal dynamics and inter-subject heterogeneity}

Figure 1 illustrates 15 min of continuous MHC tracings, CWT projection, and global wavelet power spectrum (GWPS) for 2 patients in the cohort (cardiac arrest and sepsis). MHC signal demonstrates biological variability and complexity, and the pattern of MHC variability is not similar for these patients. Using CWT, the time-dependent changes in MHC power at various frequencies is illustrated, and the CWT projections are distinctive between subjects. The cardiac arrest MHC tracing demonstrates a combination of fast and slow frequency components with no discernable pattern throughout the recording; this is reflected in the GWPS with balanced distribution of power throughout the microvascular bands. By contrast, the sepsis MHC tracing is dominated by a slow, periodic and persistent oscillation with occasional episodes of correction-a phenomenon that has been described as vasomotion [26]. In the GWPS, this is represented by a singular broad peak in the neurogenic frequency band.

Median power, \% Power, and \% CV for MHC signal for the 31 patients in the final dataset is reported in Fig. 2. Median power values in the cohort follow a lognormal distribution ( $p>0.05$, D'Agostino Pearson test). MHC signal demonstrates substantial temporal variability, represented by the $\% \mathrm{CV}$ of signal power versus time (Fig. 2b). Correlation matrix for the frequency bands is shown in supplementary Fig. 2; microvascular frequency bands B2-B4 are more closely correlated with each other than the respiratory band B5. There was no significant difference in signal power between SDD $15 \mathrm{~mm}$ and $20 \mathrm{~mm}$ (4.957 vs 4.733, $\mathrm{p}=0.2019)$.

The \% Power composition of MHC signal for each patient is shown in Fig. 3 and is not consistent across 
A

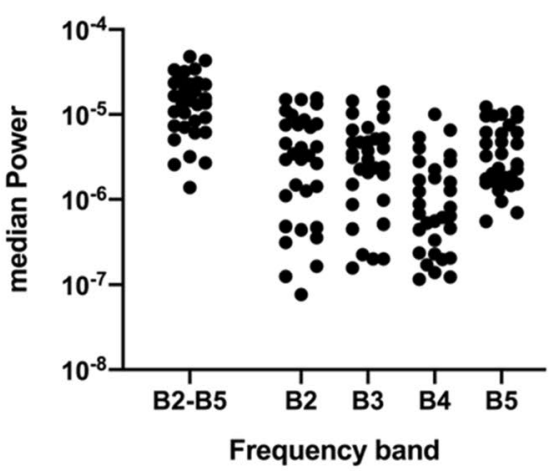

B

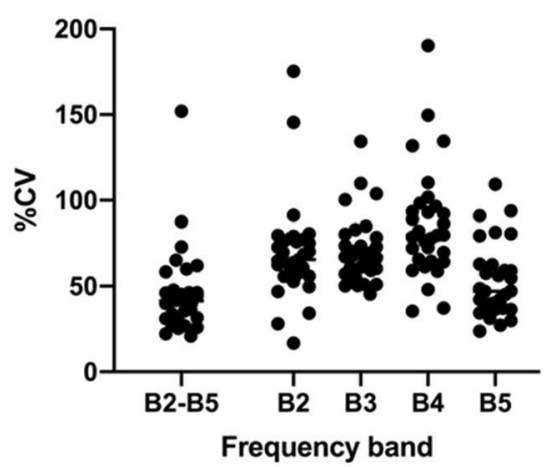

C

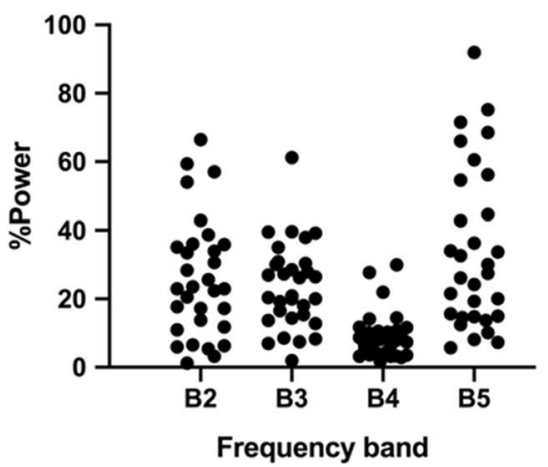

Fig. 2 MHC signal characteristics for the patients included in the final cohort $(\mathrm{n}=31$ patients), reported for total signal B2-B5 and for each frequency band ( $B 2$ endothelial, B3 neurogenic, $B 4$ myogenic, $B 5$ respiratory). a MHC median power values for patients in the cohort follows lognormal distribution ( $\mathrm{p}>0.05$, D’Agostino Pear- son test). b Temporal variation in each frequency band, expressed as coefficient of variation (\%CV) of signal power vs time. c Percentage contribution (\% Power) for each frequency band relative to total signal power B2-B5, calculated as (median Power band / median Power B2-B5) the cohort. There is varying contribution to the overall MHC power from the microvascular bands B2-B4 versus respiratory band B5 (Fig. 3a). Furthermore, the proportions of each band within the microvascular signal B2-B4 is not consistent (Fig. 3b); some patients have balanced power between all three bands, while others have two or even one frequency component that dominates the signal composition.

\subsection{MHC signal characteristics are concordant between muscle groups in the same patient and positively correlated with systemic hemoglobin and oxygenation levels}

Pairwise comparison for signal power between muscle sites in the same patient demonstrates moderate correlation for microvascular bands B2-B4 (Supplementary Figure $\mathrm{S} 3 ; \mathrm{r}=0.52-0.68, \mathrm{p}<0.05)$, but not for respiratory B5. \% Power was moderately correlated between muscle sites in all frequency bands (Supplementary Figure $\mathrm{S} 4 ; \mathrm{r}=0.48-0.73, \mathrm{p}<0.05)$. MHC microvascular power B2-B4 is positively correlated with systemic hemoglobin levels (Supplementary Figure $S 5 a ; R^{2}=0.24, p=0.0055$ ) and with $\mathrm{SpO}_{2}$ (Supplementary Figure $\mathrm{S} 5 \mathrm{~b} ; \mathrm{R}^{2}=0.15$, $\mathrm{p}=0.0410)$.

\subsection{Mechanical ventilation directly impacts MHC in peripheral tissue}

Respiratory frequency from the ventilator was readily detectable with MHC monitoring in this cohort. The effect of mechanical ventilation on MHC signal is demonstrated with $\Delta \mathrm{OD}$ time series for three patients (Fig. 4): the respiratory frequency is superimposed on the intrinsic variability of each MHC tracing. Similarly, CWT projection (Fig. 1) identifies the ventilator effect as a distinct power stripe in the respiratory band B5.

Linear regression shows a positive correlation between Power B5 and tidal volume (Supplementary Figure S6a; $\left.\mathrm{R}^{2}=0.22, \mathrm{p}=0.0141\right)$, but no correlation with PEEP or driving pressure $(\mathrm{p}>0.05)$. \% Power B5 is positively correlated with PEEP (Supplementary Figure $S 6 b ; R^{2}=0.25$, $p=0.0065)$, but no correlation with tidal volume or driving pressure $(p>0.05)$. These findings persisted with multivariate regression (supplementary Table 2).

\subsection{MHC signal power is differentiated according to vasopressor status, but not mean arterial pressure or lactate}

Vasopressor use was required in 20/31 patients (64.5\%) in the cohort. Patients receiving vasopressors had significantly lower MHC signal power B2-B5 when compared to patients not receiving vasopressors (Fig. 5a; 5.023 vs 4.715, p=0.0286), but there was no correlation between signal power and MAP (Fig. $5 b ; R^{2}=0.02, p=0.4384$ ). There was no difference in temporal variability $(\% \mathrm{CV})$ of $\mathrm{MHC}$ signal between vasopressor groups (46.1 vs 38.6, $\mathrm{p}=0.8870$ ). Median serum lactate in the cohort was 1.4 (IQR $1.0-1.8) \mathrm{mmol} / \mathrm{L}$, with $6 / 31$ patients (19.4\%) having lactate values greater than $2.0 \mathrm{mmol} / \mathrm{L}$. Serum lactate was not correlated with MHC signal power $\left(R^{2}=0.04\right.$, $\mathrm{p}=0.2600$ ), and there was no difference in signal power between patients with lactate greater or less than $2.0 \mathrm{mmol} / \mathrm{L}$ (5.133 vs 4.991, $\mathrm{p}=0.5591$ ). 

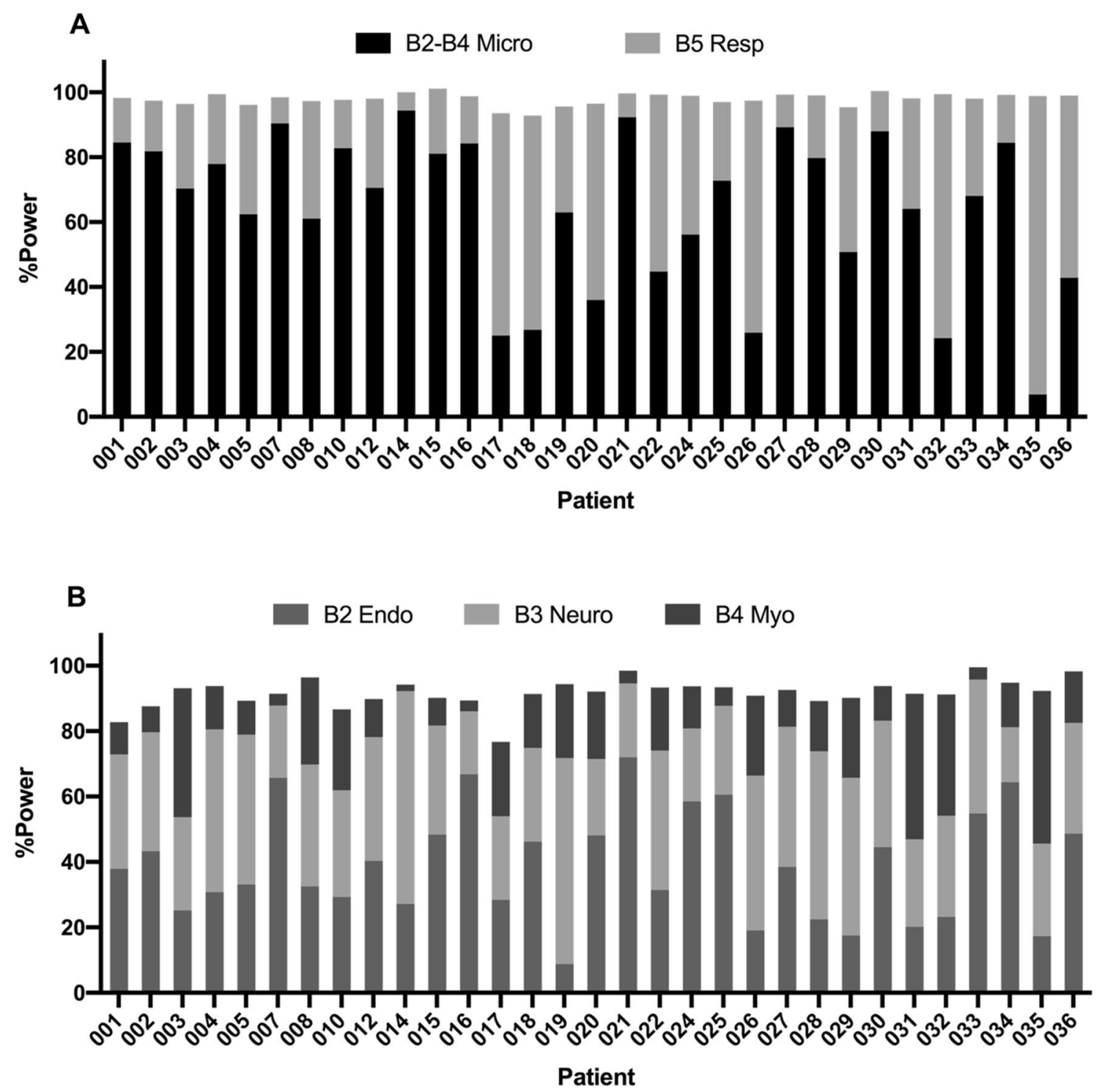

Fig. 3 Signal power composition for each patient $(n=31)$ demonstrates the percentage contribution (\% Power) from each frequency band relative to overall signal power ( $B 2$ endothelial, $B 3$ neurogenic, $B 4$ myogenic, $B 5$ respiratory). \% Power composition is not consistent between patients in the cohort. a \% Power for microvascular bands

\section{Discussion}

This pilot study demonstrates that MHC monitoring in the ICU can provide continuous physiological data for assessing peripheral perfusion. Using high-resolution data sampling and a straightforward optical device, a signal with substantial biological variability was measured. Fundamentally, this variability underscores the physiology of RBC distribution, and should be viewed as the primary method by which oxygen delivery is regulated in the microcirculation [27]. Moreover, this is the first application of CWT to NIRS data from
B2-B4 and respiratory band B5 relative to overall signal B2-B5; \%Power is calculated as (median Power/median Power B2-B5). b $\%$ Power for each microvascular band relative to microvascular signal B2-B4; \%Power is calculated as (median Power/median Power B2B4)

peripheral tissue in the ICU, representing a novel method for quantifying microvascular hemodynamics.

The main goal of this pilot study was to assess feasibility for MHC monitoring in the ICU, that was successfully demonstrated by signal acquisition from the large majority of patients in the cohort; the number of recruited patients that were invalidated was low, and for reasons that are easily corrected. Moreover, MHC signal quality was high, with a large majority of recorded data suitable for analysis. Motion artifacts were notable, but can been minimized in the future, as with other continuous physiological waveforms including pulse oximetry [28], cardiac telemetry [29], and arterial 


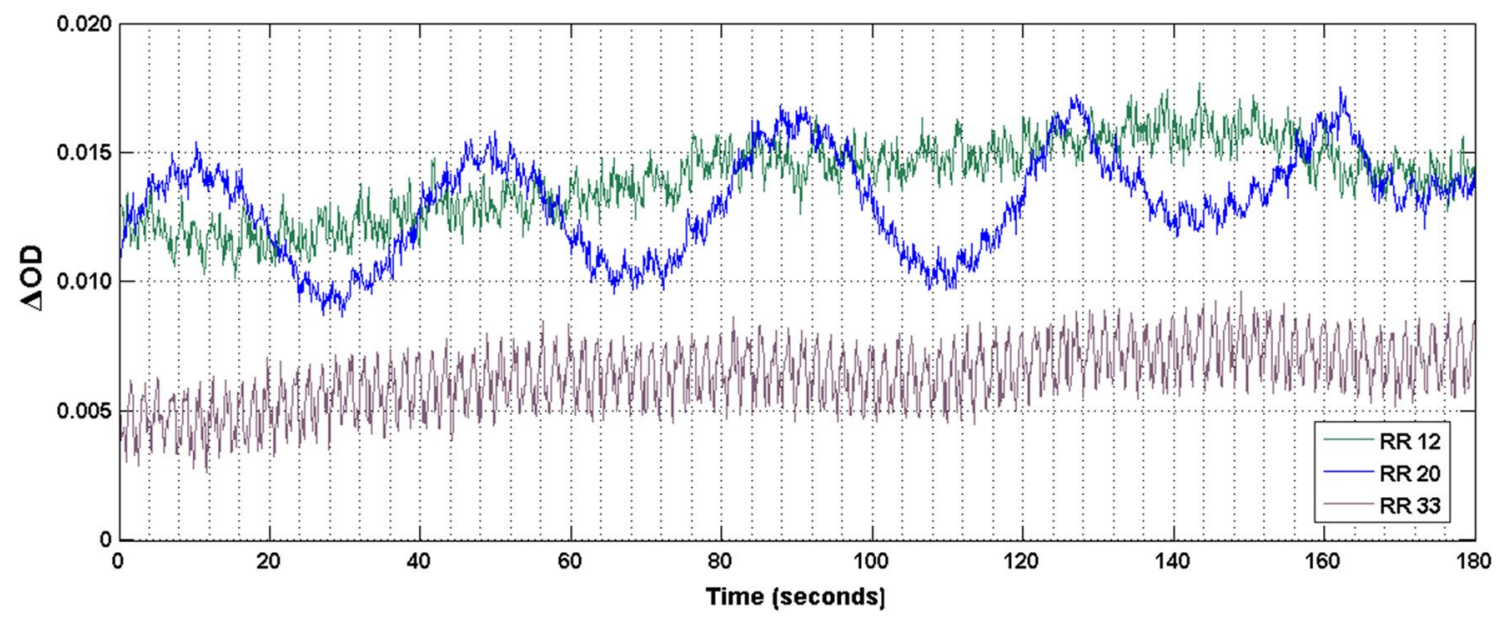

Fig. 4 High-resolution NIRS demonstrates the interaction between mechanical ventilation and MHC signal in peripheral tissue. MHC time series $(\triangle \mathrm{OD}$, arbitrary units) for three patients are shown. The mechanical ventilator is evident as a distinctive "saw-tooth" pattern superimposed on the intrinsic variability of the MHC signal. The frequency corresponds to the respiratory rate (RR, breaths per minute) delivered by the mechanical ventilator

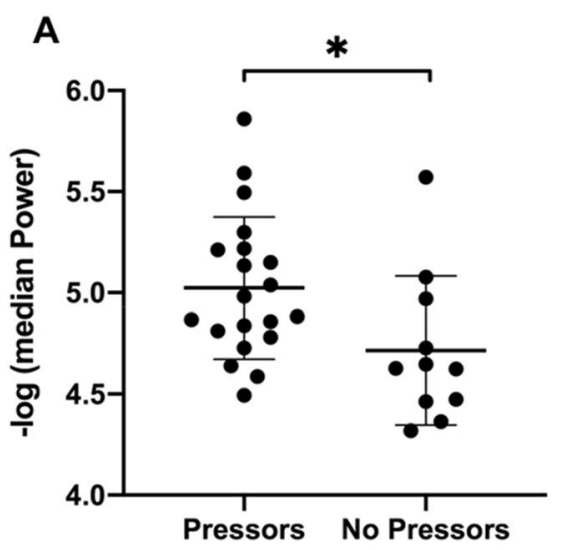

Fig. 5 MHC signal power is differentiated according to vasopressor status, but is not correlated with mean arterial pressure. Note the -logtransform data where lower values indicate higher MHC signal power. MAP mean arterial pressure. a MHC signal power B2-B5 is

blood pressure [30]. Overall, this pilot study supports ongoing clinical investigation of MHC monitoring.

Tools for quantifying peripheral perfusion in the ICU have emerged in recent decades, as technology has made bedside evaluation of the microcirculation more accessible. Sublingual microcirculation has been evaluated extensively using handheld videomicroscopy [31], and alterations to the microvascular perfusion characteristics are seen following ICU interventions [32-35], and correlated with adverse ICU outcomes [36, 37]. Dynamic assessments of peripheral perfusion using LDF [38, 39] or StO2-derived vascular occlusion tests $[40,41]$ also correlate with ICU outcomes,
B

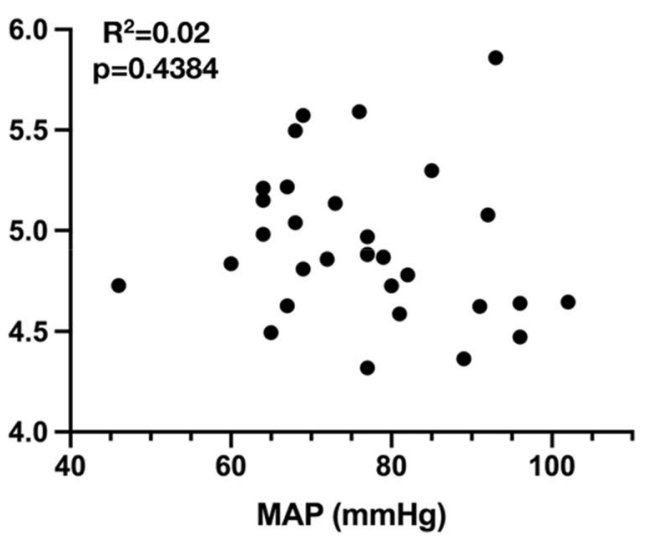

significantly reduced for patients receiving vasopressors $(* \mathrm{p}=0.0286$, Student's unpaired t-test). Lines represent mean and standard deviation. b MHC signal power B2-B5 is not correlated with mean arterial pressure

signifying the importance of the microcirculation in the pathophysiology of critical illness.

The strength of this current study is the simple application of this non-invasive optical technology, that may facilitate the adoption of MHC for monitoring deep tissue perfusion in many clinical settings. MHC is measured with NIRS using one or two wavelengths and well-accepted biomedical optics equations; the only requirement is high temporal resolution. MHC is recorded at the bedside in a similar fashion to cardiac telemetry and pulse oximetry, and is operator independent. In contrast, laser doppler and sublingual systems monitor only superficial tissue beds, and require training for data collection and analysis; they are also not conducive 
to continuous real-time monitoring in a challenging ICU environment.

This pilot study introduces MHC as a novel continuous hemodynamic metric with substantial biological variability and signal complexity (Fig. 1). These patterns of variability relate to the spatial and temporal complexity of the cardiovascular system that integrates signals from central, local, and neurogenic mechanisms. Time-frequency analysis using CWT highlights the presence of coupled oscillators that produce MHC time series with non-stationary and nonlinear dynamics $[42,43]$. These features are evident with high-resolution MHC data sampled at $10 \mathrm{~Hz}$, but are often overlooked when measuring $\mathrm{StO}_{2}$ with commercial NIRS systems that report values every $2-5 \mathrm{~s}$. Furthermore, given the slower change of $\mathrm{StO}_{2}$ in skeletal muscle (i.e. regional venous oxygen saturation), $\mathrm{StO}_{2}$ functions mostly as a trend monitor over minutes to hours rather than second-to-second variability as observed with MHC.

MHC analysis in Fig. 3 indicates that the signal composition (\% Power from each frequency band) is not consistent between patients, and that physiological heterogeneity may exist regarding peripheral perfusion characteristics; this is not surprising in a cohort of patients with complex and diverse ICU diseases. Sub-classification according to physiological profile has been proposed for sepsis using organ failure models [44, 45], lactate kinetics [46], body temperature [47], and continuous vital signs [48, 49]. These subclasses (endotypes) have identified groups with differing mortality risks and treatment response profiles. By incorporating signal from the peripheral microcirculation, $\mathrm{MHC}$ may eventually delineate physiological endotypes of perfusion that are not evident with macro-hemodynamic monitors.

This study suggests that MHC signal power may discriminate between patients according to vasopressor status but not according to MAP (Fig. 5). This is consistent with prior studies that noted vasopressor use was a better surrogate for microvascular injury than MAP or cardiac index [36]. MHC power was lower for patients receiving vasopressors, and may reflect reduced oxygen delivery consistent with computational models [50]. The relationship between systemic hemodynamics and the microcirculation is disrupted in critical illness [51], and disparities often exist between macrohemodynamics and the degree of microvascular injury [37, 52]. The absence of correlation between MAP and MHC is consistent with the concept of the microcirculation as a distinct hemodynamic compartment, and the inability for systemic parameters to predict microvascular status. The lack of correlation between $\mathrm{MHC}$ and lactate may reflect the fact that most patients had normal lactate levels $(<2.0 \mathrm{mmol} / \mathrm{L})$ at the time of monitoring, but also the differential kinetics between lactate clearance and microvascular perfusion [53]. These findings support the application of microvascular monitoring to enhance the hemodynamic management of patients with circulatory failure.

The interaction between mechanical ventilation and the circulatory system is well-described for the macrocirculation, but rarely has this impact been considered for the microcirculation. Using high-resolution NIRS, this study is the first to describe this phenomenon in skeletal muscle (Fig. 4). Respiratory-induced intensity variation with mechanical ventilation has been demonstrated with cerebral NIRS to measure regional venous saturation [54], and with pulse oximetry as a marker of volume responsiveness [55]. It is postulated that positive pressure decreases venous return and causes expansion of blood volume in the peripheral venous compartment $[54,56]$. Measured here in another vascular bed, these findings support the hypothesis that mechanical ventilation is exerting a systemic impact on the microcirculation of many organs throughout the body. MHC monitoring directly quantifies the magnitude of hemoglobin content displaced by mechanical ventilation in peripheral microvascular networks, that will depend on the interaction between ventilator parameters, pulmonary mechanics, and venous hemodynamics (e.g. central venous pressure, blood volume, flow, capacitance). At this stage, it is unclear whether this phenomenon has any direct consequences on organ function, or the mechanisms through which these consequences could be mediated.

MHC signal is comparable in different muscle groups for the same patient (supplementary Figs. 3 and 4), suggesting a connection between central and peripheral blood flow control mechanisms throughout the body. Some of the concordance between muscle groups will arise from central signals that impact the entire system (e.g. heart rate, mean arterial pressure), but there are also semi-centralized mechanisms that operate throughout vascular networks (e.g. sympathetic tone). B2 endothelial and B3 myogenic bands are ascribed to local mechanisms of blood flow control, that may be similarly affected by systemic critical illness across the spatially distributed peripheral compartment.

Systemic hemoglobin and oxygenation levels are positively correlated with MHC microvascular signal power (Supplementary Figure S5), although these findings should not be overstated in a pilot cohort. It is well known from preclinical studies that the 'network Fahreaus effect' explains the reduction in microvascular hematocrit compared with systemic hematocrit [57]; however, the relationship between systemic and microvascular hematocrit during interventions (e.g. experimental hemodilution) is not always straightforward [58]. In translational human studies, a NIRS-derived index of tissue hemoglobin was not correlated with systemic hemoglobin [59], and RBC transfusion has a variable impact on NIRS and sublingual microvascular perfusion [60, 61]. Because MHC measures the temporal change in hemoglobin content within the underlying tissue, it may track better 
with RBC flux and systemic hemoglobin levels. Reduction in signal power with decreasing arterial oxygen saturation may reflect a microvascular system with diminished physiological reserve [62]. These relationships and the possible impact of RBC transfusion on MHC signal require further exploration.

\subsection{Study limitations and future considerations}

The main technical limitation for the NIRS system used in the study is the contribution from superficial tissue to MHC signal with SDD $15 \mathrm{~mm}$ and $20 \mathrm{~mm}$, and the variable depth of tissue penetration with these configurations; similarly adipose tissue thickness was not directly measured, but attempts were made to minimize this source of error with patient selection $(\mathrm{BMI}<35)$ and probe positioning. Superficial contamination is a concern with NIRS, where discrepancies in oxygen metabolism and blood flow can exist between skin, adipose, and skeletal muscle [8]. Between these three tissues, skeletal muscle comprises the largest vascular bed with the greatest contribution to MHC variability. In this cohort, there was no difference for MHC signal power between the two SDD configurations, and CWT coherence is noted in prior studies for simultaneous measurements from skin with laser doppler and skeletal muscle with NIRS [63, 64]. This suggests MHC variability may be a more uniform metric across tissue layers, and supports the application of CWT in the current study. The decision to shorten SDD in this study was driven by technical considerations relating to sensitivity of the spectrometer, rather than overall concerns with the application of the technology. The contribution and coherence of MHC signal from superficial and deep layers of peripheral tissue can be further characterized with spatiallyresolved NIRS in future system iterations.

As illustrated with CWT projection in Fig. 1, there is a clear demarcation of the respiratory band B5, yet the boundaries between microvascular frequency bands B2-B4 used from previous studies seem arbitrary; the GWPS also shows that the spectral peaks for these oscillators can fall outside of their intended band. Although these frequency components have been defined in previous studies and ascribed to regulatory systems [21,25], their origins are proposed from indirect evidence and the underlying physiology of these oscillations is not well established [26]. Furthermore, some MHC tracings had a detectable heart rate, although this was not a consistent finding for most patients or recordings (data not shown). Overall, the results of this pilot study suggest that further work is needed to understand the true origins of these oscillations, how to measure them in the peripheral microcirculation, and how best to represent them using time-frequency projections.

Building on the findings of this pilot study, more work is needed to characterize the interactions between MHC and systemic hemodynamics. Using high-resolution NIRS, correlations between MHC and MAP can be investigated as continuous physiological waveforms, similar to the analysis developed for cerebral autoregulation [13]; systemic hemodynamic assessment can also include echocardiography or other non-invasive measures of cardiac output. Moreover, controlled studies can determine the effect of hemodynamic interventions (e.g. fluid bolus, MAP changes) on MHC signal. This current study demonstrates the impact of mechanical ventilation on peripheral tissue, and suggests that ventilator parameters may influence signal characteristics (Supplementary Figure S6). Future work should relate MHC signal to the hemodynamic effects of mechanical ventilation that have been used as a marker of fluid responsiveness (e.g. pulse pressure variation) [65].

Most importantly, while this pilot study demonstrates the feasibility of MHC monitoring in the ICU, it does not evaluate the efficacy of MHC as a diagnostic or prognostic tool. Given the substantial between-patient heterogeneity observed in this cohort, larger sample sizes will be required with the addition of healthy volunteers as a comparison group; MHC signal complexity also suggests that the best metrics for characterizing microvascular dysfunction are not yet established. Furthermore, studies have shown that microvascular monitoring may not discriminate between survivors and non-survivors during initial evaluation, and that metrics will only separate later in ICU stay $[41,66]$. Thus, the prognostic ability of MHC monitoring is best evaluated with daily assessment to correlate MHC signal with ICU outcomes. This information is a pre-requisite before any consideration for incorporating MHC as a resuscitation target. Overall, the findings from this pilot study have provided vital information regarding the nature of this novel hemodynamic signal, and will allow better refinement of system engineering, monitoring strategy, and the ability to successfully test numerous hypotheses in future studies.

\section{Conclusions}

Microvascular hemoglobin content in peripheral tissue can be measured continuously in the intensive care unit with high-resolution NIRS and reflects the dynamic variability of hemoglobin distribution in the microcirculation. Mechanical ventilation directly impacts MHC in peripheral tissue. Results suggest that this novel hemodynamic metric should be further evaluated for diagnosing microvascular dysfunction and monitoring peripheral perfusion.

Acknowledgements The authors thank Dr. Alison Fox-Robichaud for reviewing the manuscript on behalf of the Canadian Critical Care Translational Biology Group. 
Author contributions CGE: Conceptualization. AAM, CGE, MD: Study Design. CGE, AAM, MD, DB, KSL, MS: Investigatio. MD, AR, KSL, AAM: Resources (hardware). AAM, TB: Data acquisition. AAM, CGE: Data analysis. AAM: Writing — original draft. All: Writing-edit and final approval.

Funding CGE received a Collaborative Health Research Project Grant (\#RN248497) from the Canadian Institutes of Health Research and the Natural Sciences and Engineering Research Council of Canada.

Data availability The datasets generated during and/or analysed during the current study are available from the corresponding author on reasonable request.

Code availability The custom software used during the current study is available from the corresponding author on reasonable request.

\section{Compliance with ethical standards}

Conflicts of interest The authors declare no conflicts of interest.

Consent to participate Written informed consent was obtained from the participant or substitute decision maker for all individuals included in the study.

Consent to publish Written informed consent was obtained from the participant or substitute decision maker for publication of their data.

Ethical approval This study was performed in line with the principles of the Declaration of Helsinki. All research practices were performed in accordance with institutional ethics protocol and approval at Western University and Lawson Health Research Institute (REB \#108760, March 2017).

\section{References}

1. Vincent JL, Ince C, Bakker J. Clinical review: circulatory shockan update: a tribute to Professor Max Harry Weil. Crit Care. 2012;16:1-5.

2. Ait-Oufella H, Lemoinne S, Boelle PY, Galbois A, Baudel JL, Lemant J, et al. Mottling score predicts survival in septic shock. Intensive Care Med. 2011;37:801-7.

3. Dumas G, Lavillegrand JR, Joffre J, Bigé N, De-Moura EB, Baudel JL, et al. Mottling score is a strong predictor of 14-day mortality in septic patients whatever vasopressor doses and other tissue perfusion parameters. Crit Care. 2019;23:1-9.

4. Hernández G, Ospina-Tascón GA, Damiani LP, Estenssoro E, Dubin A, Hurtado J, et al. Effect of a resuscitation strategy targeting peripheral perfusion status vs serum lactate levels on 28-day mortality among patients with septic shock: the ANDROMEDA-SHOCK randomized clinical trial. JAMA. 2019;321:654-64.

5. Hariri G, Joffre J, Leblanc G, Bonsey M, Lavillegrand JR, Urbina $T$, et al. Narrative review: clinical assessment of peripheral tissue perfusion in septic shock. Ann Intensive Care. 2019;9:1-9. https://doi.org/10.1186/s13613-019-0511-1.

6. Lima A, Bakker J. Noninvasive monitoring of peripheral perfusion. Appl Physiol Intensive Care Med Physiol Rev Ed. 2012;2:39-49.
7. Dubin A, Henriquez E, Hernández G. Monitoring peripheral perfusion and microcirculation. Curr Opin Crit Care. 2018;24:173-80.

8. Barstow TJ. Understanding near infrared spectroscopy and its application to skeletal muscle research. J Appl Physiol. 2019;126:1360-76. https://doi.org/10.1152/japplphysiol.00166 .2018 .

9. Mesquida J, Gruartmoner G, Espinal C, Mesquida J, Gruartmoner $\mathrm{G}$, Espinal C. Skeletal muscle oxygen saturation (StO2) measured by near-infrared spectroscopy in the critically Ill patients. Biomed Res Int. 2013;2013:1-8. https://doi.org/10.1155/2013/502194.

10. Nardi O, Zavala E, Martin C, Nanas S, Scheeren T, Polito A, et al. Targeting skeletal muscle tissue oxygenation $\left(\mathrm{StO}_{2}\right)$ in adults with severe sepsis and septic shock: a randomised controlled trial (OTO-StS Study). BMJ Open. 2018;8:e017581. https://doi. org/10.1136/bmjopen-2017-017581.

11. Bakker A, Smith B, Ainslie P, Smith K. Near-Infrared spectroscopy. Appl Asp Ultrason Hum. 2012;2012:65-88. https://doi. org/10.5772/32493.

12. Lee JK, Kibler KK, Benni PB, Easley RB, Czosnyka M, Smielewski $\mathrm{P}$, et al. Cerebrovascular reactivity measured by near-infrared spectroscopy. Stroke. 2009;40:1820-6. https://doi. org/10.1161/STROKEAHA.108.536094.

13. Zweifel C, Dias C, Smielewski P, Czosnyka M. Continuous timedomain monitoring of cerebral autoregulation in neurocritical care. Med Eng Phys. 2014;36:638-45. https://doi.org/10.1016/j. medengphy.2014.03.002.

14. Pries AR, Secomb TW. Blood flow in microvascular networks. Microcirculation. 2008;2008:3-36. https://doi.org/10.1016/B9780-12-374530-9.00001-2.

15. Fantini S. Dynamic model for the tissue concentration and oxygen saturation of hemoglobin in relation to blood volume, flow velocity, and oxygen consumption: Implications for functional neuroimaging and coherent hemodynamics spectroscopy (CHS). Neuroimage. 2014;85:202-21. https://doi.org/10.1016/j.neuro image.2013.03.065.

16. Kim JG, Xia M, Liu H. Extinction coefficients of hemoglobin for near-infrared spectroscopy of tissue. IEEE Eng Med Biol Mag. 2005;24:118-21. https://doi.org/10.1109/MEMB.2005.1411359.

17. Davis ML, Barstow TJ. Estimated contribution of hemoglobin and myoglobin to near infrared spectroscopy. Respir Physiol Neurobiol. 2013;186:180-7. https://doi.org/10.1016/j.resp.2013.01.012.

18. Diop M, Wright E, Toronov V, Lee T-Y, Lawrencece KSt. Improved light collection and wavelet de-noising enable quantification of cerebral blood flow and oxygen metabolism by a lowcost, off-the-shelf spectrometer. J Biomed Opt. 2014;19:057007. https://doi.org/10.1117/1.JBO.19.5.057007.

19. Scholkmann F, Kleiser S, Metz AJ, Zimmermann R, Mata Pavia $\mathrm{J}$, Wolf $\mathrm{U}$, et al. A review on continuous wave functional nearinfrared spectroscopy and imaging instrumentation and methodology. Neuroimage. 2014;85:6-27. https://doi.org/10.1016/j.neuro image.2013.05.004

20. Stefanovska A, Bracic M, Kvernmo HD. Wavelet analysis of oscillations in the peripheral blood circulation measured by laser Doppler technique. IEEE Trans Biomed Eng. 1999;46:1230-9. https ://doi.org/10.1109/10.790500.

21. Kvandal P, Landsverk SA, Bernjak A, Stefanovska A, Kvernmo HD, Kirkebøen KA. Low-frequency oscillations of the laser Doppler perfusion signal in human skin. Microvasc Res. 2006;72:120-7.

22. Bernjak A, Stefanovska A. Importance of wavelet analysis in laser Doppler flowmetry time series. Annu Int Conf IEEE Eng Med Biol Proc. 2007;11:4064-7406.

23. Bernjak A, Clarkson PBM, McClintock PVE, Stefanovska A. Low-frequency blood flow oscillations in congestive heart failure 
and after beta1-blockade treatment. Microvasc Res. 2008;76:22432. https://doi.org/10.1016/j.mvr.2008.07.006.

24. Geyer MJ, Jan YK, Brienza DM, Boninger ML. Using wavelet analysis to characterize the thermoregulatory mechanisms of sacral skin blood flow. J Rehabil Res Dev. 2004;41:797-805.

25. Folgosi-Correa MS, Nogueira GEC (2011) Quantifying lowfrequency fluctuations in the laser Doppler flow signal from human skin. In: Tuchin VV, Duncan DD, Larin KV, Leahy MJ, Wang RK, editors. Proc SPIE 2011. p. 789811. Doi: https://doi. org/10.1117/12.874080

26. Pradhan RK, Chakravarthy VS. Informational dynamics of vasomotion in microvascular networks: a review. Acta Physiol. 2011;201:193-218.

27. Ellis CG, Jagger J, Sharpe M. The microcirculation as a functional system. Crit Care. 2005;9(4):S3-8. https://doi.org/10.1186/cc375 1.

28. Pollreisz D, TaheriNejad N. Detection and removal of motion artifacts in PPG signals. Mob Networks Appl. 2019. https://doi. org/10.1007/s11036-019-01323-6.

29. Patel SI, Souter MJ, Warner DS, Warner MA. Equipment-related electrocardiographic artifacts. Anesthesiology. 2008;108:138-48. https://doi.org/10.1097/01.anes.0000296537.62905.25.

30. Li Q, Mark RG, Clifford GD. Artificial arterial blood pressure artifact models and an evaluation of a robust blood pressure and heart rate estimator. Biomed Eng Online. 2009;8:13. https://doi. org/10.1186/1475-925X-8-13.

31. Ince C, Boerma EC, Cecconi M, De Backer D, Shapiro NI, Duranteau J, et al. Second consensus on the assessment of sublingual microcirculation in critically ill patients: results from a task force of the European Society of Intensive Care Medicine. Intensive Care Med. 2018;44:281-99.

32. Jhanji S, Stirling S, Patel N, Hinds CJ, Pearse RM. The effect of increasing doses of norepinephrine on tissue oxygenation and microvascular flow in patients with septic shock. Crit Care Med. 2009;37:1961-6.

33. Dubin A, Pozo MO, Casabella CA, Pálizas F, Murias G, Moseinco $\mathrm{MC}$, et al. Increasing arterial blood pressure with norepinephrine does not improve microcirculatory blood flow: a prospective study. Crit Care. 2009;13:1-8.

34. Pranskunas A, Koopmans M, Koetsier PM, Pilvinis V, Boerma EC. Microcirculatory blood flow as a tool to select ICU patients eligible for fluid therapy. Intensive Care Med. 2013;39:612-9.

35. Ospina-Tascon G, Neves AP, Occhipinti G, Donadello K, Büchele $\mathrm{G}$, Simion D, et al. Effects of fluids on microvascular perfusion in patients with severe sepsis. Intensive Care Med. 2010;36:949-55. https://doi.org/10.1007/s00134-010-1843-3.

36. Hernandez G, Boerma EC, Dubin A, Bruhn A, Koopmans M, Edul VK, et al. Severe abnormalities in microvascular perfused vessel density are associated to organ dysfunctions and mortality and can be predicted by hyperlactatemia and norepinephrine requirements in septic shock patients. J Crit Care. 2013;28:538.

37. De Backer D, Donadello K, Sakr Y, Ospina-Tascon G, Salgado $\mathrm{D}$, Scolletta S, et al. Microcirculatory alterations in patients with severe sepsis: Impact of time of assessment and relationship with outcome. Crit Care Med. 2013;41:791-9.

38. Bourcier S, Joffre J, Dubée V, Preda G, Baudel J-LL, Bigé N, et al. Marked regional endothelial dysfunction in mottled skin area in patients with severe infections. Crit Care. 2017;21:155. https:// doi.org/10.1186/s13054-017-1742-x.

39. Mongkolpun W, Orbegozo D, Cordeiro CPR, Franco CJCS, Vincent J-L, Creteur J. Alterations in skin blood flow at the fingertip are related to mortality in patients with circulatory shock. Crit Care Med. 2020;48:443-50. https://doi.org/10.1097/CCM.00000 00000004177.

40. Neto AS, Pereira VGM, Manetta JA, Espósito DC, Schultz MJ. Association between static and dynamic thenar near-infrared spectroscopy and mortality in patients with sepsis. J Trauma Acute Care Surg. 2014;76:226-33. https://doi.org/10.1097/ TA.0b013e3182a9221f.

41. Donati A, Damiani E, Domizi R, Scorcella C, Carsetti A, Tondi S, et al. Near-infrared spectroscopy for assessing tissue oxygenation and microvascular reactivity in critically ill patients: a prospective observational study. Crit Care. 2016;20:1-10. https://doi. org/10.1186/s13054-016-1500-5.

42. Glass L. Synchronization and rhythmic processes in physiology. Nature. 2001;410:277-84. https://doi.org/10.1038/35065745.

43. Stefanovska A. Coupled oscillators. Complex but not complicated cardiovascular and brain interactions. IEEE Eng Med Biol Mag. 2010;26:25-9.

44. Zhang Z, Zhang G, Goyal H, Mo L, Hong Y. Identification of subclasses of sepsis that showed different clinical outcomes and responses to amount of fluid resuscitation: a latent profile analysis. Crit Care. 2018;22:347. https://doi.org/10.1186/s1305 4-018-2279-3.

45. Gårdlund B, Dmitrieva NO, Pieper CF, Finfer S, Marshall JC, Taylor Thompson B. Six subphenotypes in septic shock: latent class analysis of the PROWESS Shock study. J Crit Care. 2018;47:70-9.

46. Thomas-Rueddel DO, Poidinger B, Weiss M, Bach F, Dey K, Häberle $\mathrm{H}$, et al. Hyperlactatemia is an independent predictor of mortality and denotes distinct subtypes of severe sepsis and septic shock. J Crit Care. 2015;30:439.e1-439.e6. https://doi. org/10.1016/j.jcrc.2014.10.027.

47. Bhavani SV, Carey KA, Gilbert ER, Afshar M, Verhoef PA, Churpek MM. Identifying novel sepsis subphenotypes using temperature trajectories. Am J Respir Crit Care Med. 2019;200:327-35. https://doi.org/10.1164/rccm.201806-1197OC.

48. Kamaleswaran R, Akbilgic O, Hallman MA, West AN, Davis RL, Shah SH. Applying artificial intelligence to identify physiomarkers predicting severe sepsis in the PICU. Pediatr Crit Care Med. 2018;19:e495-503. https://doi.org/10.1097/PCC.0000000000 001666.

49. van Wyk F, Khojandi A, Mohammed A, Begoli E, Davis RL, Kamaleswaran R. A minimal set of physiomarkers in continuous high frequency data streams predict adult sepsis onset earlier. Int J Med Inform. 2019;122:55-62. https://doi.org/10.1016/j.ijmed inf.2018.12.002.

50. Fraser GM, Sharpe MD, Goldman D, Ellis CG. Impact of incremental perfusion loss on oxygen transport in a capillary network mathematical model. Microcirculation. 2015;22:348-59.

51. Ince $\mathrm{C}$. Hemodynamic coherence and the rationale for monitoring the microcirculation. Crit Care. 2015;19:S8. https://doi org/10.1186/cc14726.

52. Kanoore Edul VS, Ince C, Vazquez AR, Rubatto PN, Valenzuela Espinoza ED, Welsh S, et al. Similar microcirculatory alterations in patients with normodynamic and hyperdynamic septic shock. Ann Am Thorac Soc. 2016;13:240-7.

53. Hernandez G, Pedreros C, Veas E, Bruhn A, Romero C, Rovegno $\mathrm{M}$, et al. Evolution of peripheral vs metabolic perfusion parameters during septic shock resuscitation. A Clin Study J Crit Care. 2012;27:283-8. https://doi.org/10.1016/j.jcrc.2011.05.024.

54. Wolf M, Duc G, Keel M, Niederer P, Von Siebenthal K, Bucher HU. Continuous noninvasive measurement of cerebral arterial and venous oxygen saturation at the bedside in mechanically ventilated neonates. Crit Care Med. 1997;25:1579-82.

55. Nilsson LM. Respiration signals from photoplethysmography. Anesth Analg. 2013;117:859-65. https://doi.org/10.1213/ ANE.0b013e31828098b2.

56. Leung TS, Tisdall MM, Tachtsidis I, Smith M, Delpy DT, Elwell CE. Cerebral tissue oxygen saturation calculated using low frequency haemoglobin oscillations measured by near infrared spectroscopy in adult ventilated patients. Adv Exp Med Biol. 
2008;614:235-44. https://doi.org/10.1007/978-0-387-74911 $-2 \_27$.

57. Pries AR, Ley K, Gaehtgens P. Generalization of the Fahraeus principle for microvessel networks. Am J Physiol. 1986;251:H1324-32. https://doi.org/10.1152/ajphe art.1986.251.6.H1324.

58. Pries AR, Fritzsche A, Ley K, Gaehtgens P. Redistribution of red blood cell flow in microcirculatory networks by hemodilution. Circ Res. 1992;70:1113-21. https://doi.org/10.1161/01. res.70.6.1113.

59. Doerschug KC, Delsing AS, Schmidt GA, Haynes WG. Impairments in microvascular reactivity are related to organ failure in human sepsis. Am J Physiol Circ Physiol. 2007;293:H1065-71. https://doi.org/10.1152/ajpheart.01237.2006.

60. Sadaka F, Aggu-Sher R, Krause K, O’Brien J, Armbrecht ES, Taylor RW. The effect of red blood cell transfusion on tissue oxygenation and microcirculation in severe septic patients. Ann Intensive Care. 2011;1:46. https://doi.org/10.1186/2110-5820-1-46.

61. Scheuzger J, Zehnder A, Meier V, Yeginsoy D, Flükiger J, Siegemund M. Sublingual microcirculation does not reflect red blood cell transfusion thresholds in the intensive care unit-a prospective observational study in the intensive care unit. Crit Care. 2020;24:18. https://doi.org/10.1186/s13054-020-2728-7.
62. Seely AJE, Macklem P. Fractal variability: an emergent property of complex dissipative systems. Chaos. 2012;22:013108. https:// doi.org/10.1063/1.3675622.

63. Thorn CE, Shore AC, Matcher SJ (2007) Combined optical and near infrared reflectance measurements of vasomotion in both skin and underlying muscle. In: Chance B, Alfano RR, Tromberg BJ, Tamura M, Sevick-Muraca EM, editors. Opt Tomogr Spectrosc Tissue VII 2007. p. 643421. https://doi.org/https://doi. org/10.1117/12.700801

64. Yano T, Lian CS, Afroundeh R, Shirakawa K, Yunoki T. Comparison of oscillations of skin blood flow and deoxygenation in vastus lateralis in light exercise. Biol Sport. 2014;31:15-20.

65. Monnet X, Marik PE, Teboul JL. Prediction of fluid responsiveness: an update. Ann Intensive Care. 2016;6:1-11.

66. Sakr Y, Dubois M-J, De Backer D, Creteur J, Vincent J-L. Persistent microcirculatory alterations are associated with organ failure and death in patients with septic shock*. Crit Care Med. 2004;32:1825-31. https://doi.org/10.1097/01.CCM.0000138558 $.16257 .3 \mathrm{~F}$.

Publisher's Note Springer Nature remains neutral with regard to jurisdictional claims in published maps and institutional affiliations. 